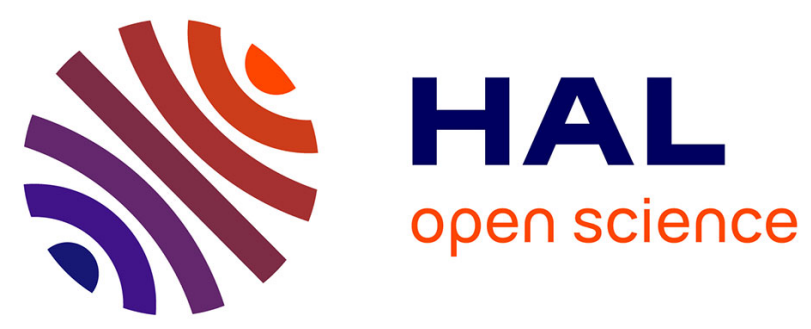

\title{
LEONAR liner concept: multiphysics coupling in presence of grazing flow, thermal gradients and high sound levels
}

Victor Lafont, Fabien Mery, Frank Simon

\section{- To cite this version:}

Victor Lafont, Fabien Mery, Frank Simon. LEONAR liner concept: multiphysics coupling in presence of grazing flow, thermal gradients and high sound levels. AIAA Aviation 2021 Forum, Aug 2021, Virtual event, United States. pp.AIAA 2021-2204, 10.2514/6.2021-2204 • hal-03416243

\author{
HAL Id: hal-03416243 \\ https://hal.science/hal-03416243
}

Submitted on 5 Nov 2021

HAL is a multi-disciplinary open access archive for the deposit and dissemination of scientific research documents, whether they are published or not. The documents may come from teaching and research institutions in France or abroad, or from public or private research centers.
L'archive ouverte pluridisciplinaire HAL, est destinée au dépôt et à la diffusion de documents scientifiques de niveau recherche, publiés ou non, émanant des établissements d'enseignement et de recherche français ou étrangers, des laboratoires publics ou privés. 


\title{
LEONAR liner concept: multiphysics coupling in presence of grazing flow, thermal gradients and high sound levels
}

\author{
Victor Lafont* and Fabien Méry ${ }^{\dagger}$ \\ ONERA/DMPE - Université de Toulouse, F-31055, Toulouse, France \\ Frank Simon \\ ONERA/DMPE - Université de Toulouse, F-31055, Toulouse, France
}

\begin{abstract}
The development of turbofans with larger fan diameters, shorter inlets, and thinner walls forces the acoustic liners to be placed closer to the hot parts of engines. This experimental study investigates the combined effects of important thermal gradients, grazing flow and acoustic level on the acoustic behavior of liners applied to the LEONAR (Long Elastic Open Neck Acoustic Resonator) liner concept. Previous studies have shown that a coupling between these three effects can exist. The objective is thus to understand the underlying coupled phenomena, in order to emphasize the interest of such a liner concept regarding a classical equivalent SDOF liner.

In the ONERA B2A grazing flow acoustic liner facility, the flow temperature can be accurately regulated and several types of acoustic excitation can be provided. A test section with a heating or cooling device is used to obtain a thermal gradient between the backplate and the perforated plate of the liner sample, and infrared (IR) thermography is used to measure the temperature distribution on the perforated plate. The measurement is conducted on several configurations and with different types of liners, to assess the behaviors of the LEONAR liner concept in the context of the UHBR.
\end{abstract}

\section{Nomenclature}

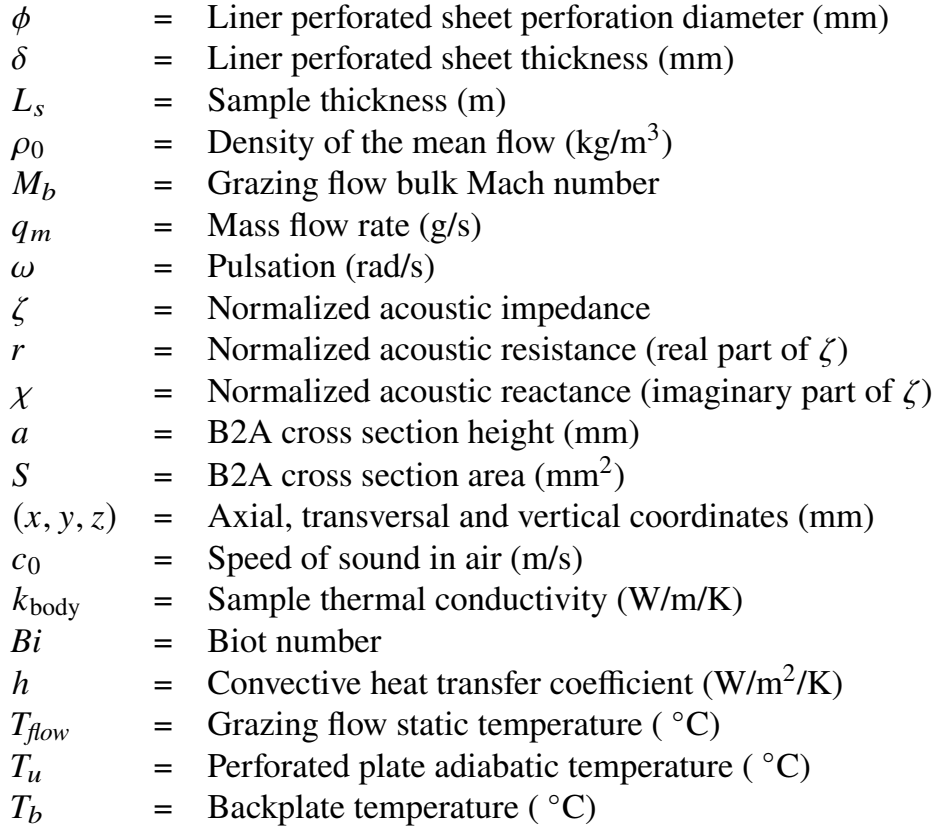

\footnotetext{
*Phd student, victor.lafont@onera.fr

$\dagger$ Research Scientist, fabien.mery@onera.fr

$\doteqdot$ Research Scientist, frank.simon@onera.fr
} 


\section{Introduction}

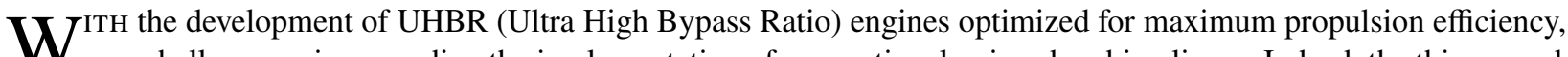
new challenges arise regarding the implementation of conventional noise-absorbing liners. Indeed, the thinner and shorter nacelles will leave less room to accommodate acoustic liners, whereas these same liners need to increase in height in order to properly absorb the lower-frequency noise generated by a larger fan. Moreover, the thinner walls between the combustion chamber and the bypass ducts will be likely to induce thermal gradients inside the liners' structure itself. Therefore, there is a growing need to propose new liner concepts that deal with these challenges. These concepts must be characterized as precisely as possible and their behavior must be assessed when thermal gradients, complex flows and high noise levels are involved simultaneously.

Conventional, single degree of freedom (SDOF) liners consist of a honeycomb structure topped with a thin perforated facesheet, forming a layout of small resonators closed at their bottom by a rigid backplate. The geometry of the honeycomb can be adjusted to match specific noise damping requirements [1]. The driving parameter for the noise damping power of "locally reacting" liners is their acoustic impedance, a complex number that is the ratio between acoustic pressure and normal acoustic velocity taken on the facesheet, normalized by the impedance of air $\rho_{0} c_{0}$ :

$$
\zeta(\omega)=\frac{Z(\omega)}{\rho_{0} c_{0}}=\frac{p}{\rho_{0} c_{0} v_{n}}=r(\omega)+\mathrm{j} \chi(\omega)
$$

where $r$ and $\chi$ are respectively called the resistance and the reactance of the liner. It is generally admitted that resistance can be increased by the sound pressure level (SPL) or by the grazing flow. These effects are so-called nonlinear effects linked to a vortex shedding produced periodically from both ends of each perforation. Having a varying resistance due to SPL could be a drawback in terms of designing liner solution. Several model exists and take into account these effects. Guess [2] for instance gave a solution to model the effect of SPL and grazing flow. It appears necessary to propose a solution that is as much as possible weakly nonlinear, for example, with a thicker perforated plate so the nonlinear effects are slightly reduced (because of a high ratio between the perforated plate thickness $\delta$ and the hole diameter $\phi$ [3, 4]). This solution is obviously simple and acoustically efficient but the thicker plate cannot be an acceptable solution in terms of mass. In the same idea, the total height of the cavity is a driving parameter for the frequency selection: to absorb at lower frequencies, a higher cavity is required. Again, this is not fully compatible with aeronautical constraints in the UHBR context. The LEONAR concept (or HREN for Helmholtz Resonator with Extended Neck) [5, 6] could be a good candidate to cope with these challenges. It consists in linking the perforated layer with hollow tubes introduced in the honeycomb, to shift resonance frequencies to lower frequencies by a prolongation of air column lengths. Recently, Guo et al. [7] proposed the design of an optimal liner constructed by 16 inhomogeneous HRENs which enables to have a sound absorption in a prescribed frequency range from 700 to $1000 \mathrm{~Hz}$. The same authors applied this concept to a small-scale propeller [8] where the liner concept should be very compact regarding the available space. These types of liner concepts are thus very interesting in order to design the optimal liner for a given application.

The coupling between aerodynamic and acoustic effects is complex, as liners increase the viscous drag in the turbulent layer compared to a smooth surface. Howerton et al. [9] investigated the drag effect of several conventional or more advanced liner designs in the NASA GFIT. They found that the resistance factor $\lambda$ (also known as the friction factor) was frequency-dependent in presence of acoustic excitation. This effect increased with sound pressure level, but was mitigated by higher flow speeds. More recently, Jasinski et al. [10] highlighted that a dramatic increase in drag at frequencies near acoustic resonance and at high sound levels is observed on classical SDOF liners. Zhang and Bodony [11] confirmed that high acoustic levels increased the liner drag by performing a series of Direct Numerical Simulations (DNS) reproducing the conditions of a single orifice-over-cavity liner undergoing a grazing flow. Leon et al. [12] used a high-magnification PIV setup to obtain 2D acoustic velocity fields measured close to the perforations of a SDOF liner sample. They observed that high acoustic levels induced specific aerodynamic phenomena and that the "rough-wall" analysis was showing its limits to predict the effects. Regarding the thermal effects, Elnady et al. [13] investigated the acoustic behavior of a single orifice over a cavity placed in an oven. The surface of the sample was hotter than the back of the cavity, the difference was up to $100 \mathrm{~K}$. The measurements were conducted for several SPL, including nonlinear regime, but without any grazing flow. The impedance of the liner was measured with an in-situ technique and compared to existing semi-empirical models. It was found that an increase in temperature results in a decrease of the reactance, while the resistance slightly increases (at least when the nonlinear effects are small compared to the viscous ones). Modifying the properties of air in the model to account for the high temperatures appeared to be enough to predict quite well this impedance change; the decrease of the reactance is indeed due to the modification of the air density which impacts the sound speed inside the cavity. 
Giachetti et al. [14] experimentally studied the influence of synthetic pulsating jets across a multi-perforated wall. They have studied the role of cross-flow and synthetic jet interactions and the effect on the convective thermal coefficient. It is the first experimental set up that enables to understand which types of coupling can be observed in the case of liners with grazing flow, acoustic excitations and thermal gradients present together. A numerical restitution of the previous experiment was proposed by Esnault [15]. Méry et al. [16] conducted a study on liner samples heated from the back wall, both with and without the presence of a cooler grazing flow. The acoustic response of the samples was investigated both in the linear and nonlinear regimes with respect to the acoustic level. They showed that the temperature changes mainly impacted the reactance as the sound speed inside the cavities was modified. They also highlighted the influence of the acoustic level on the temperature repartition: for high SPL, the temperature went down inside the cavity due to the sample entering the nonlinear regime. This highlighted strong multiphysics coupling between acoustics, flow and thermal phenomena. Especially, the nonlinear effect due to the sound pressure level on the surface temperature and the thermal exchanges have been highlighted in Lafont et al. [17]

In this study, a multiphysics assessment will be performed on a LEONAR liner concept in order to characterize this king of concept and to give ensights to use it in the UHBR context. This concept is compared to an acoustically equivalent SDOF liner. A specific experimental setup is designed and used to monitor precisely the evolution of a liner surface temperature when there is a thermal gradient inside it and in presence of high sound pressure levels. The measurements are conducted both with a hot and cool grazing flow and the experimental conditions are carefully controlled. In particular, the temperature is monitored in several places all along the measurement. Impedance eduction is performed based on [18].

The objective of this study is to highlight the differences in terms of impedance and thermal effects between two liners that should acoustically behave the same. The LEONAR concept enables to reduce the total height of a given liner concept and thus reduce the overall mass but it is important to highlight the impact of this architecture on the thermal repartition and the impedance evolution in presence of a grazing flow and a thermal gradient. The first part will be dedicated to the liner definition, the experimental methods and the setup. The second part will address impedance eduction results on these two liner concepts. Finally, thermography results on the skin surface liner will be presented and discussed. The first section of this article is dedicated to the methodology and the setup. The second section highlights the acoustic characterization of the liner sample and the final section deals with the thermal characterization results.

\section{Experimental methods and setup}

\section{A. Sample description}

Two samples are used in this study. One is a simple SDOF liner that has already been studied previously at ONERA[16]. The second one is a LEONAR, a type of liner where the perforations of the facesheet are connected to hollow tubes extending inside the cavities (see Fig. 11). This specific geometry allows to increase the perceived thickness of the facesheet without modifying the total height of the liner [6], leading to a lower-band absorption and a very linear liner with respect to incident SPL. The LEONAR sample is based on the geometry of the SDOF sample. Thus, the perceived thickness of the perforated sheet is the same but the total height is reduced. The LEONAR sample is expected to have similar acoustic characteristic but to differ in the thermal and grazing flow behavior. The two samples are metallic samples obtained by additive manufacturing.

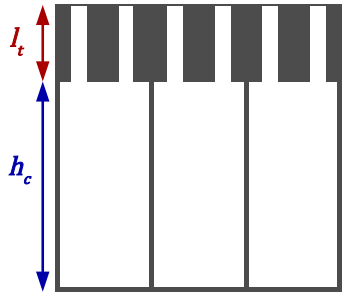

SDOF

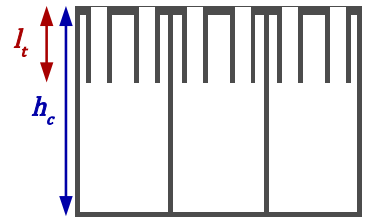

LEONAR

Fig. 1 Comparison of equivalent SDOF (left) and LEONAR (right). $h_{c}$ is the inner cavity height, $l_{t}$ the tube length $(=\delta$ for a SDOF sample) 
Table 1 Geometric characteristics of the samples

\begin{tabular}{lccccc}
\hline Sample & $\phi(\mathrm{mm})$ & Porosity $(\mathrm{POA})$ & $\delta$ or $l_{t}(\mathrm{~mm})$ & Inner cavity height $(\mathrm{mm})$ & Total height $(\mathrm{mm})$ \\
\hline SDOF & 1.5 & $15 \%$ & 8 & 41 & 50 \\
LEONAR & 1.5 & $15 \%$ & $1+7$ & 41 & 45 \\
\hline
\end{tabular}

\section{B. Acoustic setup}

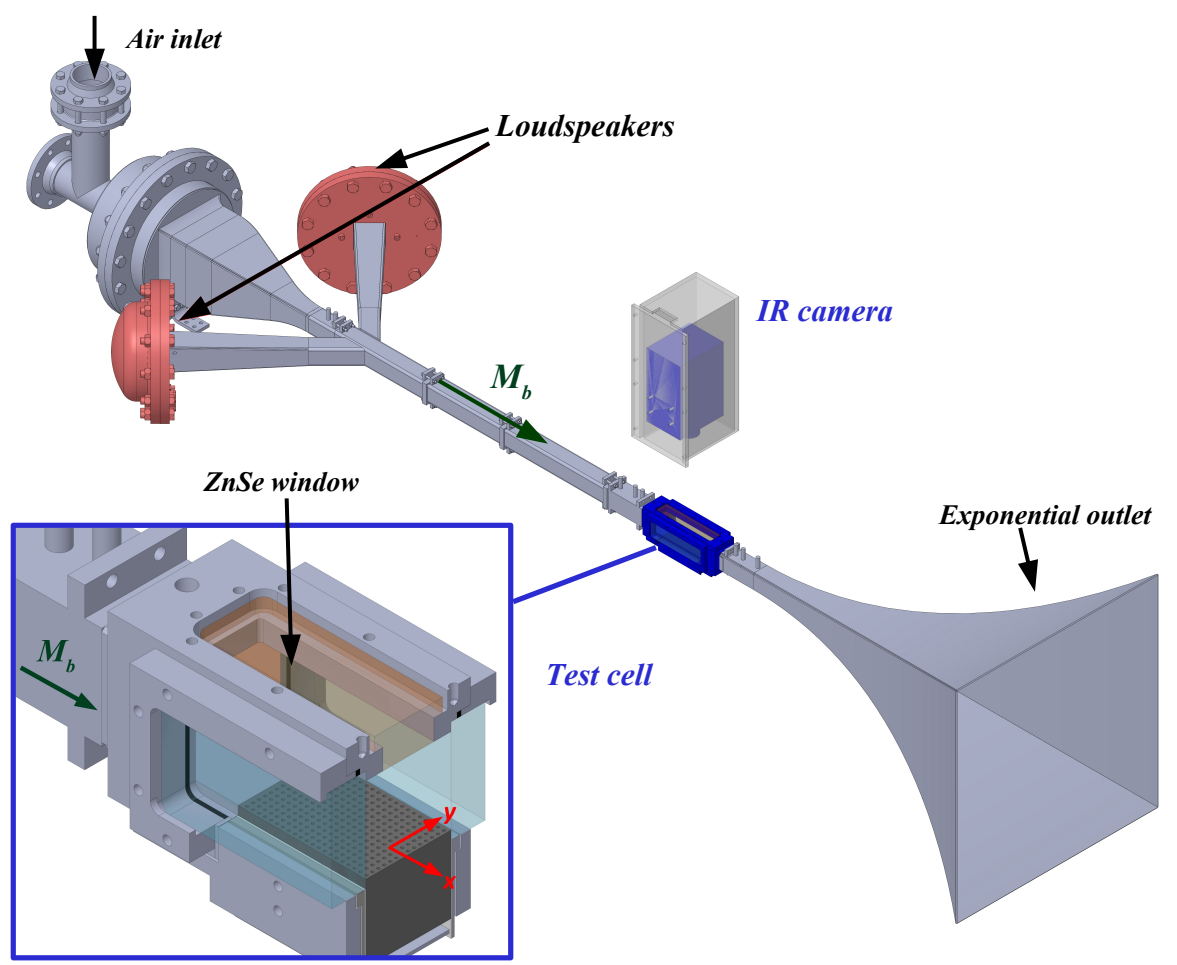

Fig. 2 The B2A bench

The B2A test bench is made of a stainless steel tube with a square section of side $a=50 \mathrm{~mm}$ and a total length of about $4 \mathrm{~m}$. A mean flow of bulk Mach number $M_{b}$ up to 0.5 can be provided, its temperature can be accurately regulated from room temperature up to $570 \mathrm{~K}$. In the duct, the flow is in a fully-developed turbulent state, with axial velocity fluctuations on the centerline being a few percent of the bulk velocity $U_{b}$ [12].

The test section is $0.2 \mathrm{~m}$-long and equipped with two opposing silica windows for optical access. An exponential quasi-anechoic outlet terminates the duct, leading to a reflection coefficient smaller than 0.2 for frequencies higher than $500 \mathrm{~Hz}$. The surface of the test liner forms a $150 \mathrm{~mm}$-long portion of the lower wall of the flow duct and spans all the duct width [19].

16 microphone sockets in the upper wall of the test section are used for acoustic measurements and impedance eduction. The upper wall with the microphone sockets is removable and can be replaced by a window for infrared measurements. Silica windows on both sides allow near-wall optical measurements (LDV or PIV).

Upstream of the test section, two speakers are used to generate tones (usually a multi-sine signal) at up to $150 \mathrm{~dB}$ over a frequency range of 0.3 to $3.5 \mathrm{kHz}$ (i.e. the no-flow cut-off frequency of the duct for plane waves). Mono-sine or multi-sine acoustic excitation can be used. The multi-sine is made of 12 pure tones ranging from $504 \mathrm{~Hz}$ to $2824 \mathrm{~Hz}$ (see Table 2) and of equal SPL, while the mono-sine is only a single pure tone. Multi-sine excitations are used to study global behaviors at low and medium SPLs while mono-sine excitations are used for studying the behavior at higher SPLs and at specific frequencies, for example near the liner's resonance. When a multi-sine source is used, the overall 
SPL (OASPL) within the duct is much higher (usually $10 \mathrm{~dB}$ more) than the SPL at each tone frequency.

Table 2 Frequencies used (the frequency used for mono-sine excitations is written in bold).

\begin{tabular}{llllllllllll}
\hline \multicolumn{10}{c}{ Frequencies $(\mathrm{Hz})$} \\
\hline 504 & 616 & 760 & $\mathbf{9 9 2}$ & 1112 & 1240 & 1488 & 1736 & 1992 & 2248 & 2488 & 2824 \\
\hline
\end{tabular}

In the duct, the flow is in a fully-developed turbulent state, with axial velocity fluctuations on the centerline being a few percent of the bulk velocity $U_{b}$.[12] The parameter we use is the mass flow rate $q_{m}=\rho M_{b} c_{0} S$ (in grams per second), where $M_{b}=\frac{U_{b}}{c_{0}}$ is the bulk Mach number, $S=a^{2}$ is the duct cross-section, and $c_{0}$ is the sound speed in the duct.

This ensures that the conditions above the sample remain similar when the flow is heated or cooled. Table 3 shows the different flow rates used in this study and the corresponding bulk Mach numbers at different temperatures.

Table 3 Flowrates used and Mach numbers depending on the flow temperature

\begin{tabular}{cccc}
\hline$q_{m}(\mathrm{~g} / \mathrm{s})$ & $M_{b}\left(T_{\text {flow }}=20^{\circ} \mathrm{C}\right)$ & $M_{b}\left(T_{\text {flow }}=87^{\circ} \mathrm{C}\right)$ & $M_{b}\left(T_{\text {flow }}=117^{\circ} \mathrm{C}\right)$ \\
\hline 50 & 0.048 & 0.054 & 0.056 \\
100 & 0.097 & 0.107 & 0.112 \\
150 & 0.145 & 0.161 & 0.168 \\
200 & 0.194 & 0.215 & 0.224 \\
260 & 0.251 & 0.278 & 0.290 \\
\hline
\end{tabular}

\section{Thermal design and setup}

Two distinct thermal configurations are chosen for our study. They are defined by the sign of the temperature difference $\Delta T=T_{b}-T_{\text {flow }}$ between the flow and the bottom of the liner. In the positive case, the bottom wall of the liner is heated and the flow at room temperature acts as a cold thermostat to obtain a thermal gradient in the sample. In the negative case, the flow is heated and the bottom wall is kept at a lower temperature to obtain a temperature gradient (Fig. 3 and 4).

To ensure these two configurations can be obtained in a stable manner, a new test cell has been designed (Fig. 57. It is partly based on the one developed for the study by Méry et al. [16] and can withstand high temperatures while remaining sealed and without undergoing significant thermal expansion. The sample holder has been designed to include as much thermal insulation as possible, so that significant thermal exchanges can only take place between the upper and lower walls of the sample and not on the four sides. The heating or cooling systems needed to obtain a thermal gradient are positioned under the sample and were designed to achieve thermal gaps between backplate and flow up to $150 \mathrm{~K}$. During the experiment, the flow temperature and the bottom temperature are monitored using thermocouples.

In the positive configuration, the bottom of the sample is heated by two electric resistors that are screwed under a thin aluminum plate positioned just under the sample. This setup ensures a homogeneous heating distribution. In addition, the junction between the aluminum plate and the sample is sealed with thermal paste to ensure a good thermal conductivity. The heating is regulated by an external system allowing to have a constant given temperature on the bottom of the sample. This system combines a PID controller and a solid-state relay and works by setting a temperature target and controlling the resistors' power input to adjust to the true temperature measured with a type $\mathrm{K}$ thermocouple placed on top of the aluminum plate.

In the negative gradient configuration, the cooling of the sample's backplate is provided by circulating water through a metal block which is positioned under the sample. As with the heating plate, thermal paste is used at the junction between the block and the sample. The temperature regulation is obtained using a thermostatic bath that maintains a precise temperature and water flow rate. The water temperature is set to around $10{ }^{\circ} \mathrm{C}$ and the ideal flow rate is determined by a $1 \mathrm{D}$ preliminary calculation. If $P_{d}$ is the power transmitted between the bottom of the sample and the outside, then:

$$
P_{d}=\frac{T_{u}-T_{b}}{L_{s} / k_{\text {body }}}=h_{\text {water }} S_{e}\left(T_{b}-T_{\text {water }}\right)
$$


where $S_{e}$ is the total exchange area between water and metal bloc and $h_{\text {water }}$ is the corresponding convection heat coefficient. The water runs through tubes of diameter $d_{t}$ drilled into the metal bloc, so $h_{\text {water }}$ can be expressed as:

$$
h_{\text {water }}=N u \frac{k_{\text {water }}}{d_{t}}
$$

where $N u$ is the Nusselt number and $k_{\text {water }}$ is the thermal conductivity of water. The value of $N u$ is obtained through Colburn's correlation for a turbulent duct flow [20]:

$$
N u=0.023 \times R e^{0.8} \times \operatorname{Pr}^{1 / 3}
$$

where $R e$ and $\operatorname{Pr}$ are respectively the Reynolds and Prandtl numbers. For temperatures around $290 \mathrm{~K}$, we use $R e=10^{4} \times v_{\text {water }}$ where $v_{\text {water }}$ is the water speed in $\mathrm{m} / \mathrm{s}$ inside the tubes. The minimal flowspeed (in $\left.\mathrm{m} / \mathrm{s}\right)$ is then given by:

$$
v_{\text {water }}=\left(\frac{P_{d}}{36.45 \times \operatorname{Pr}^{1 / 3} \times \frac{k_{\text {water }}}{d_{t}} S_{e}\left(T_{b}-T_{\text {water }}\right)}\right)^{\frac{1}{0.8}}
$$

which, as we use 3 tubes $\left(d_{t}=10 \mathrm{~mm}\right)$ along the whole length of the sample, equals to a flow rate of around 3 to 5 $\mathrm{L} / \mathrm{min}$ depending on the conditions of the air flow in the test section.

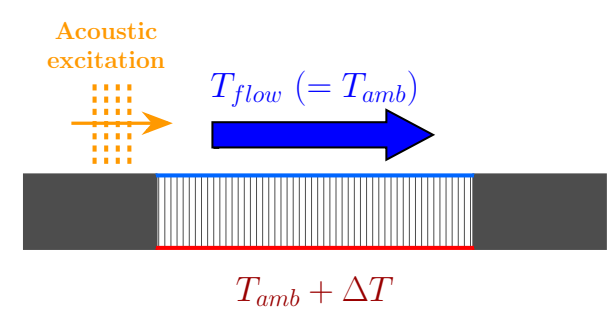

Fig. 3 Positive gradient

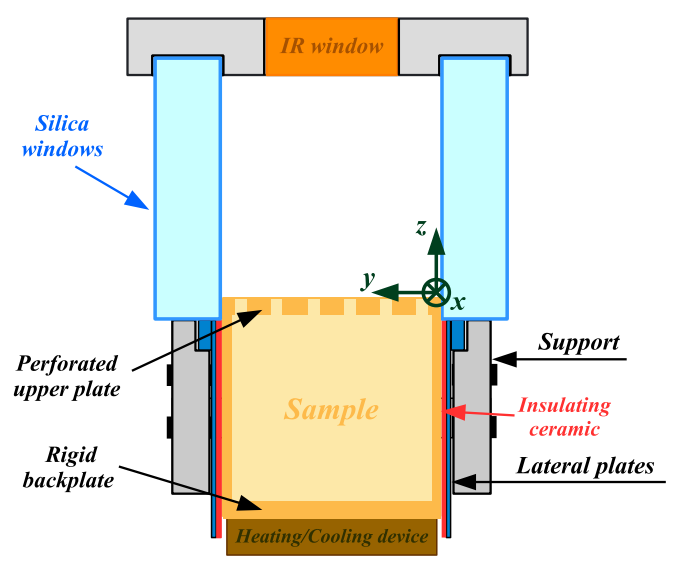

Fig. 5 Cross-section of the testing cell (facing downstream)

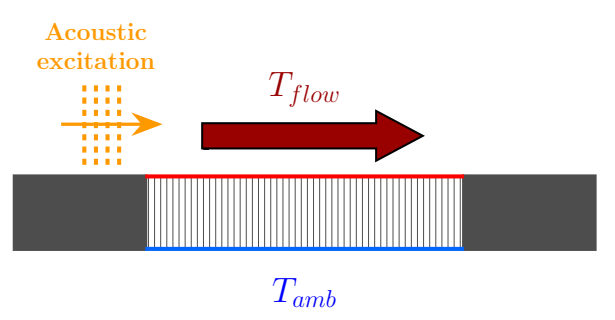

Fig. 4 Negative gradient

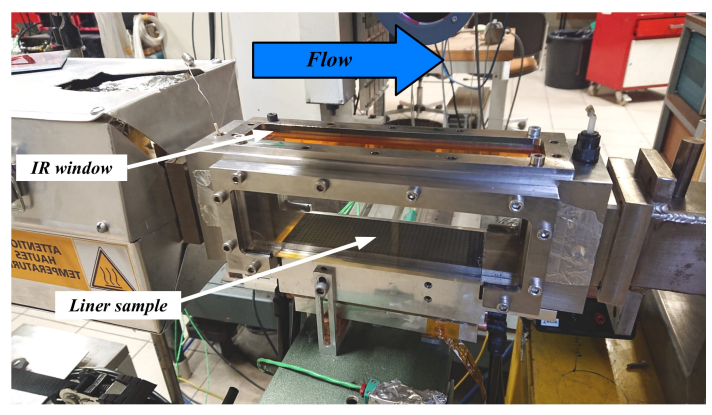

Fig. 6 Picture of the test section (the flow and $x$ axis go from left to right)

\section{Setup for infrared thermography}

The temperature of the perforated plate $T_{u}$ is obtained by infrared thermography (Fig. 6). Infrared (IR) thermography is a method of obtaining the temperature of a body by measuring the infrared radiation it emits. The technique relies on a good understanding of the physical phenomena involved and requires carefully controlled experimental conditions. An IR camera and a specific IR window with surface treatment adapted to transmit almost $100 \%$ of the incident infrared light are used. The IR window is placed in the top wall of the test section, allowing to see from above the perforated 
sheet of the sample. Two examples of raw infrared pictures are displayed in Fig. 7 and 8 . The raw pictures are 640 pixels wide and 512 pixels high. The spatial resolution is approximately 4 pixels per millimeter. The camera is set up in order to have the area of interest centered in the picture, to minimize the impact of the distortion caused by the lens at the extremities. The camera's internal calibration law is established for several specific temperature ranges. In this study, the calibration used is valid for $15{ }^{\circ} \mathrm{C}<T_{u}<73{ }^{\circ} \mathrm{C}$.

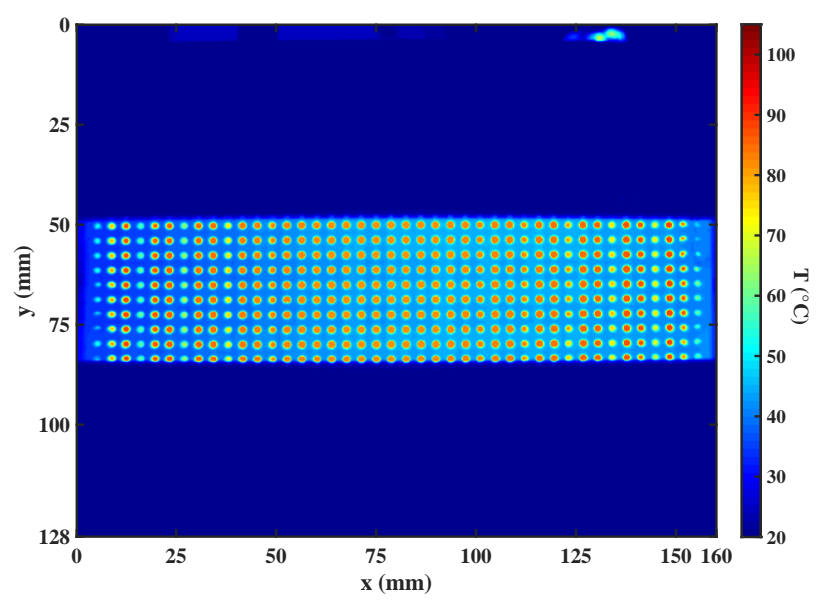

Fig. 7 Raw infrared picture, SDOF sample, $q_{m}=\mathbf{5 0} \mathrm{g} / \mathrm{s}, T_{\text {flow }}=\mathbf{2 0}{ }^{\circ} \mathrm{C}$

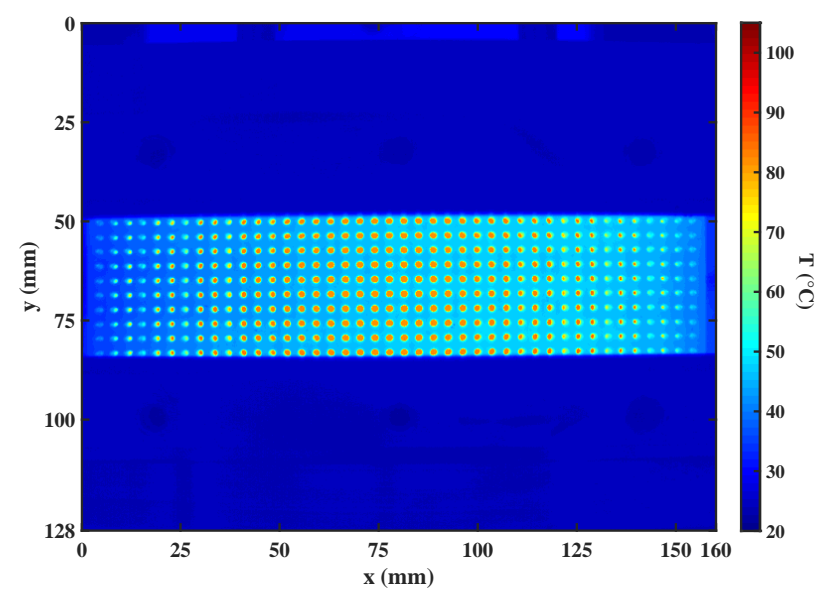

Fig. 8 Raw infrared picture, LEONAR sample, $q_{m}=\mathbf{5 0} \mathrm{g} / \mathrm{s}, T_{\text {flow }}=\mathbf{2 0}{ }^{\circ} \mathrm{C}$

An assessment of the complete setup's thermal stability was conducted using a plain rigid aluminum sample; this preliminary study is detailed in [17].

\section{Impedance and acoustic results}

\section{A. Transmission Loss}

The global behavior of the samples is determined by measuring the transmission loss between upstream and downstream sections when the samples are mounted in the B2A test cell. The transmission loss (TL, expressed in $\mathrm{dB}$ ) represents the ratio between the incident power (upstream, respective to the sound's propagation direction) and the transmitted power (downstream, respective to the sound's propagation direction), provided the downstream part ends into an anechoic termination. The incident acoustic wave (i.e. the one travelling downstream) is a multi-sine set to $130 \mathrm{~dB}$ per tone. The incident and reflected acoustic pressures are measured using a two-microphone method [16, 21]. The transmission loss (in $\mathrm{dB}$ ) is then obtained by substracting the incident pressure in the downstream section from the incident pressure in the upstream section.

The transmission losses measured on the SDOF and LEONAR samples for $q_{m}=100 \mathrm{~g} / \mathrm{s}$ are plotted in Fig. 9 and 10. The optimal absorption band of the sample is the frequency band where the transmission loss is maximal. For the SDOF and LEONAR samples, this band is located around $1 \mathrm{kHz}$. The maximal TL values of these samples are close, and the width of the band is similar as well. The acoustic behavior of the LEONAR sample is thus very similar to the behavior of the SDOF. The TL is not significantly modified in presence of a thermal gradient. The main effect appears to be a slight shift of the maximum loss band towards higher frequencies, indicating a similar change in the resonance frequency. This result is coherent with Mery et al. [16]: the shift is due to the variation of the temperature inside the cavity, which impacts directly the Helmholtz resonator characteristics.

\section{B. Impedance eduction}

The impedance of both samples is determined with an impedance eduction method based on the two-dimensional time-harmonic linearized Euler equations (LEE). This method relies on measuring the acoustic pressure on the wall opposite the liner and using a minimization method where experimental and numerical results are compared. In this study, microphone measurements were used as inputs for the eduction process. The numerical resolution of the LEE was obtained with a discontinuous Galerkin (DG) scheme, accounting naturally for the presence of a shear grazing flow 


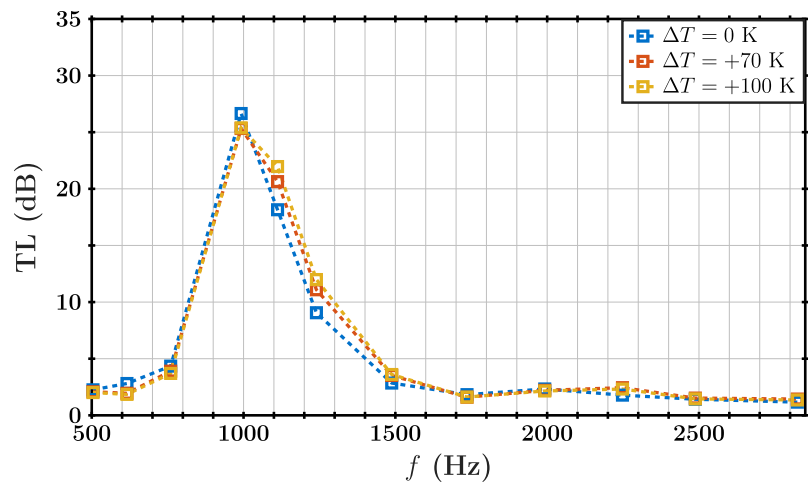

Fig. 9 Transmission Loss (TL), $q_{m}=100 \mathrm{~g} / \mathrm{s}$, sample SDOF

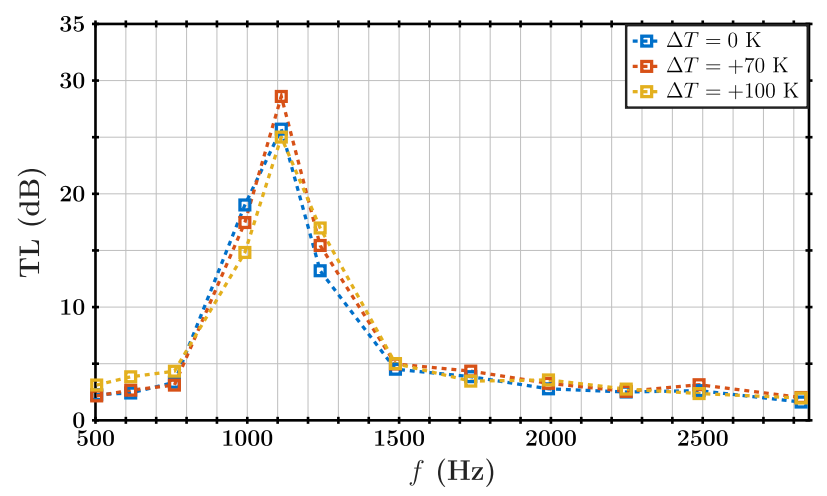

Fig. 10 Transmission Loss (TL), $q_{m}=100 \mathrm{~g} / \mathrm{s}$, sample LEONAR

in the simulation [18, 22, 23].

Figures 11 to 14 show the impedance eduction results on both samples for $q_{m}=100 \mathrm{~g} / \mathrm{s}$ and $q_{m}=260 \mathrm{~g} / \mathrm{s}$, with an incident SPL set to $130 \mathrm{~dB}$ per tone and for three thermal gradient conditions. The acoustic similarity between both samples appears clearly: their respective impedances have the same behavior over the considered frequency range, and their resonance frequencies (i.e. the frequency at which $\chi=0$ ) are very close. The influence of the grazing flow is visible and similar on both samples: the resistance $r$ increases when $q_{m}$ is greater. However, the thermal conditions do not seem to affect much the resistance and the reactance of the two liners: the only significant and consistent effect is a slight shift of the resonance towards the higher frequencies previously highlighted in [16]. This effect seems to be confirmed for the SDOF liner but is reduced on the LEONAR liner. The extended necks of the LEONAR sample apparently have an impact on the temperature distribution inside the cavities; this impact appears significant enough to counterbalance the effect of the thermal gradient on the reactance.

\section{Thermal characterization results}

For each thermal configuration and each sample, the objective is to assess the turbulent and acoustic effects by comparing the surface temperatures of the liner with and without acoustic excitation.

\section{A. Influence of the type of liner}

Figures 15 to 18 show the measured surface temperature on SDOF and LEONAR liner samples at two different flow rates, with a positive thermal gradient defined by $\Delta T=+100 \mathrm{~K}$. Three acoustic conditions are presented: without excitation, with a multi-sine excitation of SPL $=130 \mathrm{~dB} /$ tone, and with a single tone excitation near the resonance $(992 \mathrm{~Hz}$ for both samples) of SPL $=140 \mathrm{~dB}$. The profiles are taken in the streamwise direction. The asymmetry of the streamwise profiles is explained by the thermal discontinuities that result in locally higher values of the convective heat transfer coefficient $h[17]$.

The surface temperature is lower on the LEONAR than on the SDOF sample in all cases. This is explained by the differences in their internal structures which impacts their respective thermal conductivities. The equivalent thermal conductivity of the samples is indeed computed taking into account the conductivity of the material they are made of and the conductivity of the air trapped inside the cavities. A different internal geometry, such as tubes in the case of the LEONAR sample, results in a different thermal conductivity and thus a different surface temperature.

Without any acoustic excitation (blue curves), the heat convection effect at the surface is clearly visible on both samples. For each sample, when the flow rate increases, the surface temperature decreases as the heat convection at the liner surface increases. The multi-sine acoustic excitation (red curves) leads to a decrease of the surface temperature. This effect is due to the increase of heat convection at the surface as the flow and acoustic waves interact with the perforations. The reaction is a global one: the decrease is similar over virtually the whole liner length. The temperature decrease is less important when $q_{m}$ is higher, highlighting the competition between grazing flow and acoustic effects.

On the SDOF sample, when the acoustic excitation is a single tone with a frequency close to the resonance of the sample (yellow curves), the phenomenon is growing. The surface temperature decreases a lot at the beginning of the liner but then increases gradually along the liner until it matches again the value in the case with multi-sine excitation. 


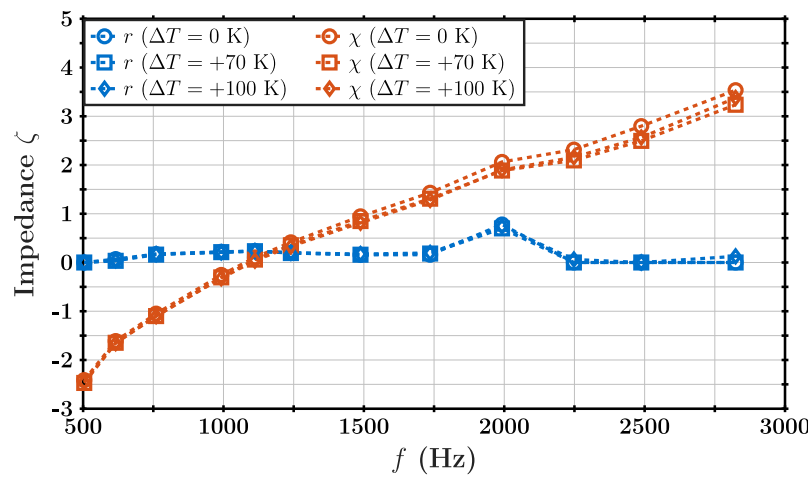

Fig. 11 LEONAR, $q_{m}=100 \mathrm{~g} / \mathrm{s}$

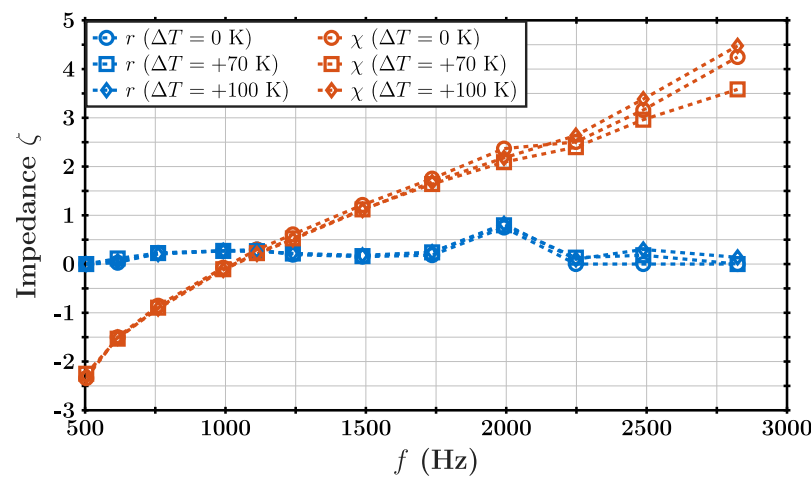

Fig. 13 SDOF, $q_{m}=100 \mathrm{~g} / \mathrm{s}$

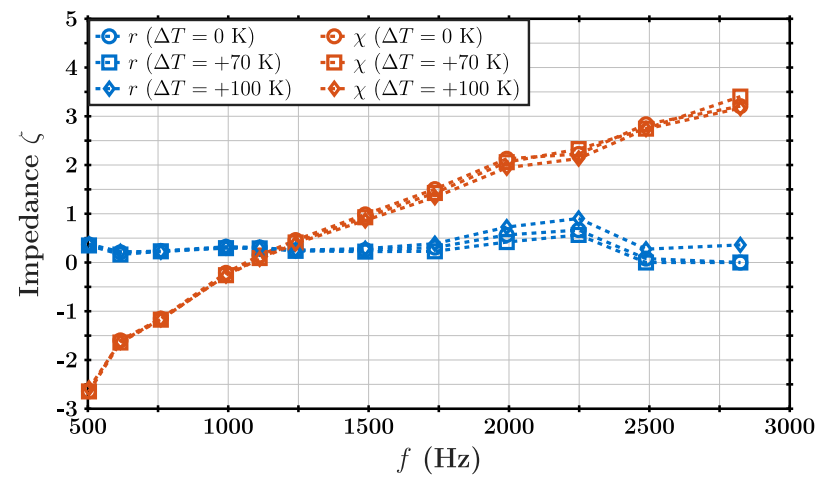

Fig. 12 LEONAR, $q_{m}=260 \mathrm{~g} / \mathrm{s}$

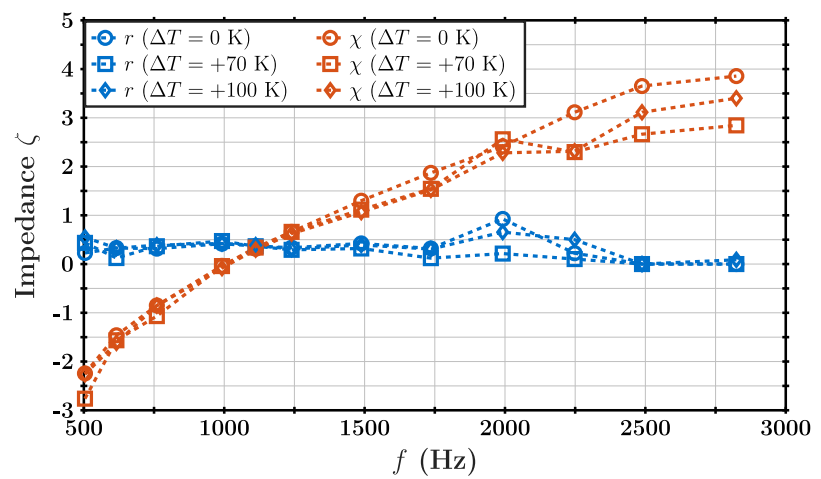

Fig. 14 SDOF, $q_{m}=260 \mathrm{~g} / \mathrm{s}$

This behavior is explained by the absorption along the sample: as the acoustic energy is absorbed, the SPL decreases and the thermo-acoustic coupling effect fades out. This is mainly due to the vortex-shedding phenomenon that increases the heat convection at the liner surface. This specific behavior near resonance does not appear as clearly on the LEONAR sample, highlighting that the thermal behavior of this sample differs from the thermal behavior of the SDOF sample. This could be explained by the differences in the internal structure. Indeed, the thermocouple measurements made by Méry et al. [16] on the same SDOF sample showed that most of the temperature gradient occurs in the first few millimeters below the perforated plate; in the case of the LEONAR, the extended necks may change this temperature distribution inside the cavities, making the vortex shedding effect much less effective as the air is trapped in the spaces between the extended necks.

\section{B. Influence of the direction of the thermal gradient}

The effects at the liner surface can also be described using the Biot number $B i$ instead of the temperature, as it allows easier comparisons between thermal configurations thanks to its link to the convective heat tranfer coefficient. The Biot number is defined by:

$$
B i=\frac{L_{s} h}{k_{\text {body }}}
$$

where $h$ is the convective heat transfer coefficient $L_{s}$ is the sample's height (in the $z$ direction) and $k_{\text {body }}$ is the computed equivalent thermal conductivity of the sample.

In our setup, solving the $1 \mathrm{D}$ heat equation yields:

$$
B i=\frac{T_{b}-T_{u}}{T_{u}-T_{\text {flow }}}
$$

where $T_{b}, T_{u}$ and $T_{\text {flow }}$ are the temperatures of the backplate, the perforated plate and the grazing flow respectively.

When the coupling between acoustics and thermal effects is even on the whole liner surface, the averaged Biot number over the whole liner surface is a good description of the phenomena involved. As shown in Fig. 19, when the 


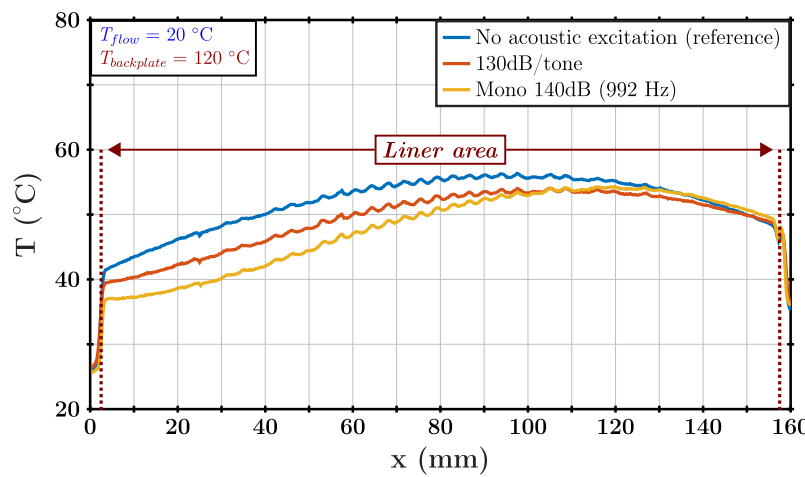

Fig. 15 Sample SDOF, $q_{m}=50 \mathrm{~g} / \mathrm{s}, \Delta T=+100 \mathrm{~K}$, different sound excitations, streamwise profile.

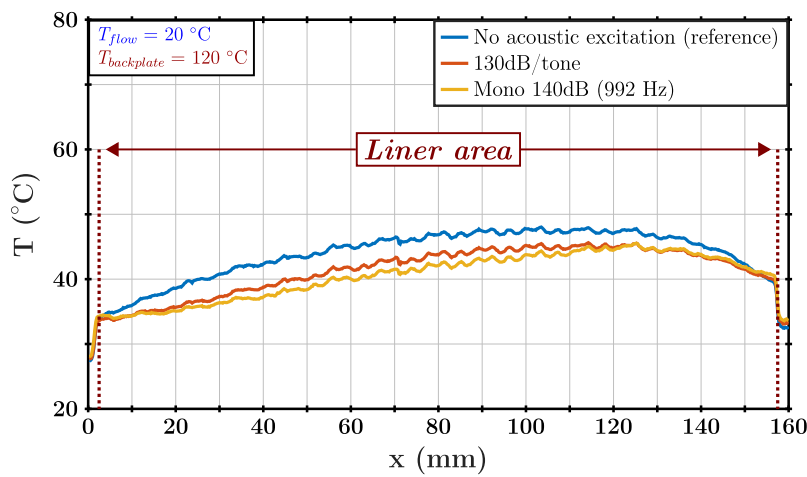

Fig. 17 Sample LEONAR, $q_{m}=\mathbf{5 0} \mathrm{g} / \mathrm{s}, \Delta T=+\mathbf{1 0 0} \mathrm{K}$, different sound excitations, streamwise profile.

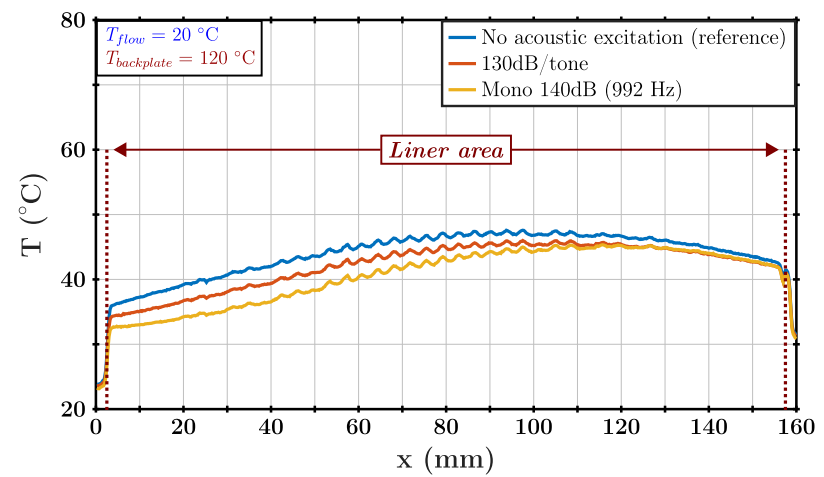

Fig. 16 SDOF, $q_{m}=100 \mathrm{~g} / \mathrm{s}, \Delta T=+100 \mathrm{~K}$, different sound excitations, streamwise profile.

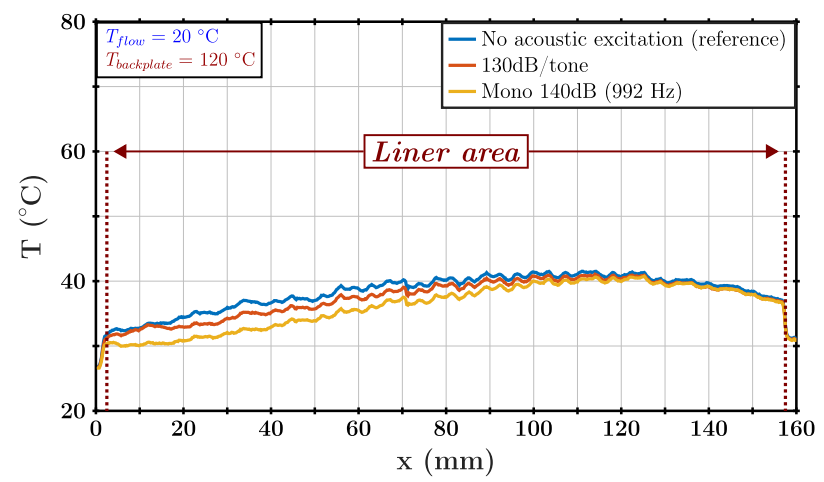

Fig. 18 LEONAR, $q_{m}=100 \mathrm{~g} / \mathrm{s}, \Delta T=+100 \mathrm{~K}$, different sound excitations, streamwise profile.

acoustic is on, the averaged Biot number increases: as all other boundary conditions remain unchanged, this means that the heat convection increases at the surface of the sample. The increase is similar on both samples, even if the reference value is not the same due to a lower surface temperature on the LEONAR sample.

The higher Biot numbers on the LEONAR sample are linked to its thermal conductivity: indeed, the model used to compute the equivalent thermal conductivities of the samples is based both on the conductivity of the material and the conductivity of the air. Both samples have roughly the same cavity volume, but the LEONAR sample has less material and that material is also less conductive than the material used for the SDOF sample, therefore the thermal conductivity of the LEONAR sample is lower than the conductivity of the SDOF sample.

Using the Biot number allows to compare directly different thermal configurations, as it eliminates the influence of the sign of the thermal gap $\Delta T: B i$ is always positive and it increases with $h$ no matter the actual temperature of the flow. Table 4 presents the values of $B i$ obtained on both samples, as well as the absolute and relative variations $\Delta B i$ and $\Delta B i / B i_{\text {ref }}$, at several flow speeds and different thermal gradient values. For both samples, $\Delta B i$ decreases when $q_{m}$ increases, no matter the value of $\Delta T$; this confirms that the grazing flow dominates the coupling effect at higher flow speeds. At a given flow speed, the values of the Biot number are identical when $\Delta T=+70 \mathrm{~K}$ and when $\Delta T=+100 \mathrm{~K}$. The same result can be observed in cases with $\Delta T<0$. This suggests that the value and direction of the gradient have little impact on the intensity of the coupling between thermal and acoustic effects.

The slight discrepancy between the values of the Biot number for positive and negative thermal gradient configurations at the same flow rate is explained by the differences between the experimental setups. Indeed, for the experiments the flow rate was taken as the reference, but it is in fact the Mach number $M_{b}$ that is linked to the intensity of the flow and thus to the intensity of the coupling. Since $M_{b}$ is higher in negative configurations due to the higher flow temperature, the flow effect is stronger and thus the increase of $B i$ is lower than in the equivalent (in terms of flow rate) positive configurations. Moreover, in the positive case, the bottom of the liner is heated but the rest of the test section stays at ambient temperature, while in the negative case the whole duct heats up with the flow; the convective effects are 
thus different. Figure 20 present the data from the last column of Table 4 , plotted as a function of the Mach number instead of the flow rate to illustrate this effect. Apart from the measurements at $q_{m}=150 \mathrm{~g} / \mathrm{s}$ which appear to be off, the general behavior is consistent no matter the thermal configuration, and the observed gaps between negative and positive gradients indeed seem to be linked to the increase of $M_{b}$ in negative thermal configurations. There is a clear gap between the behavior at low flow speeds $\left(M_{b}\right.$ smaller than 0.1$)$ and at higher flow speeds; this gap confirms that the coupling between thermal and acoustic effects is competing with the grazing flow effect. This competition between the influences of high sound levels and high flow speeds has been previously studied [23]: it was found that for Mach numbers lower than 0.1 , the acoustic effects dominate, but are gradually overwhelmed by the grazing flow until they become too small to be captured.

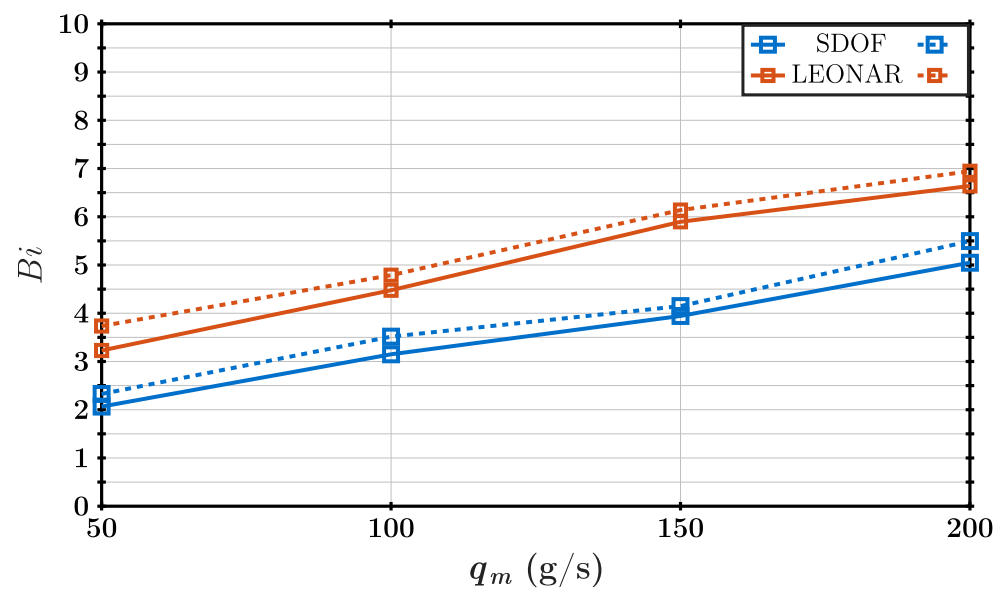

Fig. 19 Biot number (averaged over the whole surface) as a function of $q_{m}$, comparison between reference value (solid lines) and with acoustic excitation of $\mathrm{SPL}=130 \mathrm{~dB} /$ tone (dashed lines) for each sample, $\Delta T=+100 \mathrm{~K}$.

Table 4 Biot number evolution for different thermal conditions on both samples. $B i$ is averaged over the whole liner surface.

\begin{tabular}{|c|c|c|c|c|c|c|}
\hline Sample & $q_{m}(\mathrm{~g} / \mathrm{s})$ & $\Delta T$ & $B i_{r e f}$ & $B i_{130}$ & $\Delta B i$ & $\Delta B i / B i_{r e f}$ \\
\hline \multirow{10}{*}{ SDOF } & \multirow{4}{*}{50} & +100 & 2.15 & 2.59 & 0.44 & $20.5 \%$ \\
\hline & & +70 & 2.15 & 2.61 & 0.46 & $21.4 \%$ \\
\hline & & -70 & 1.6 & 1.87 & 0.27 & $16.9 \%$ \\
\hline & & -100 & 1.57 & 1.83 & 0.25 & $16.9 \%$ \\
\hline & \multirow{2}{*}{100} & +100 & 3.14 & 3.52 & 0.37 & $11.8 \%$ \\
\hline & & -100 & 2.55 & 2.71 & 0.16 & $6.3 \%$ \\
\hline & 150 & +100 & 3.94 & 4.15 & 0.21 & $5.1 \%$ \\
\hline & 200 & +100 & 5.04 & 5.49 & 0.45 & $8.9 \%$ \\
\hline & \multirow{2}{*}{260} & +100 & 6.19 & 6.39 & 0.2 & $3.2 \%$ \\
\hline & & -100 & 5.8 & 5.87 & 0.07 & $1.2 \%$ \\
\hline \multirow{6}{*}{ LEONAR } & \multirow{2}{*}{50} & +100 & 3.16 & 3.7 & 0.54 & $17.1 \%$ \\
\hline & & +70 & 3.12 & 3.76 & 0.64 & $20.5 \%$ \\
\hline & \multirow{2}{*}{100} & +100 & 4.68 & 4.95 & 0.27 & $5.8 \%$ \\
\hline & & +70 & 4.43 & 4.69 & 0.26 & $5.9 \%$ \\
\hline & 150 & +100 & 5.89 & 6.14 & 0.25 & $4.2 \%$ \\
\hline & 200 & +100 & 6.63 & 6.94 & 0.31 & $4.7 \%$ \\
\hline
\end{tabular}




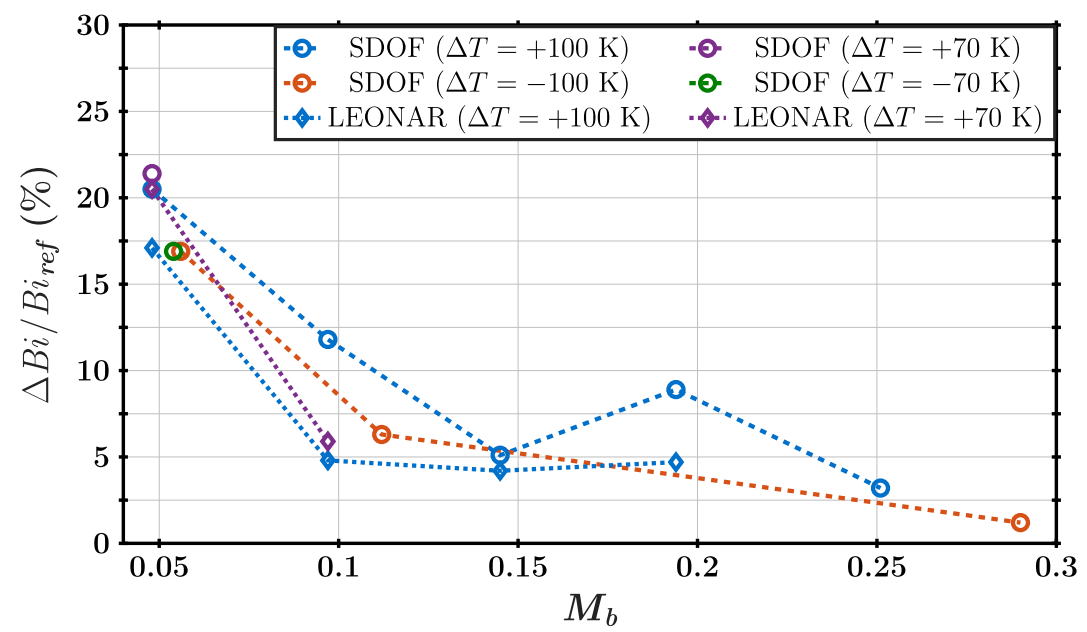

Fig. 20 Relative variations of the Biot number as a function of $M_{b}$, comparison between the two samples in different thermal configurations. SPL set to $130 \mathrm{~dB} /$ tone.

\section{Conclusion}

The behavior of two different types of liners under the influence of grazing flow, high SPL and thermal gradients has been studied using infrared thermography. Both liners are almost identical; the differences between them lie in their internal structure. Thus, their acoustic behaviors, characterized using a classical impedance eduction method, are very similar. A specific experimental setup allowed to create precise thermal gradients and to measure the samples' surface temperature with infrared thermography. The influence of these thermal gradients on the impedance of the samples was found to be small, but a coupling between high acoustic levels and the surface temperature of liners has been exhibited. This thermo-acoutic coupling is linked to an increase of the heat convection at the surface of the liner caused by the incident acoustic waves. Hence, the Biot number $B i$ was used to describe it and compare the behaviors of different liners in various conditions. The flow speed seems to be the main driving parameter for the intensity of the coupling: the additional heat convection effect is high at low flow rates but is reduced for higher flow rates due to the grazing flow effect overwhelming the acoustic level effect. This behavior is similar on both samples when the acoustic excitations are broadband, and the existence and intensity of the coupling turn out to be independent of the actual value and direction of the thermal gradient between the sample's top and bottom. The coupling effect observed is thus clearly linked to the acoustic behavior of the liners, which explains the competition with the influence of the grazing flow. Near the acoustic resonance, the thermo-acoustic coupling becomes localized; this is mainly linked to the structure and behavior of the sample with respect to high incident SPL, as it appears mostly on the SDOF sample which is less linear than the LEONAR concept regarding SPL effects. The thermo-acoustic coupling observed on these two samples is still smaller than the coupling that appears on SDOF sample with thin facesheets, which are even more nonlinear with respect to incident SPL [17]. The LEONAR sample presents an interest in the context of the UHBR since its acoustic behavior is similar to the equivalent SDOF sample and it takes less space and mass. Moreover, this study has shown that its surface temperature is less affected by incident acoustic waves near the acoustic resonance due to its specific internal structure.

\section{Acknowledgments}

The work presented in this paper has been funded by Région Occitanie. The authors thank Delphine Sebbane and Nicolas Fasano for designing and helping with the experimental setup, as well as Philippe Reulet and Thomas Batmalle for helping during the infrared measurements. Rémi Roncen is warmly acknowledged for his help on the impedance eduction part.

\section{References}

[1] Motsinger, R., and Kraft, R., "Design and performance of duct acoustic treatment," Aeroacoustics of Flight Vehicles: Theory and Practice. Volume 2: Noise Control, 1991.

[2] Guess, A., "Calculation of perforated plate liner parameters from specified acoustic resistance and reactance," Journal 
of Sound and Vibration, Vol. 40, No. 1, 1975, pp. 119-137. https://doi.org/10.1016/S0022-460X(75)80234-3, URL http://www.sciencedirect.com/science/article/pii/S0022460X75802343.

[3] Ingård, U., and Labate, S., "Acoustic circulation effects and the nonlinear impedance of orifices," The Journal of the Acoustical Society of America, Vol. 22, No. 2, 1950, pp. 211-218. https://doi.org/10.1121/1.1906591.

[4] Ingård, U., and Ising, H., "Acoustic nonlinearity of an orifice," The journal of the Acoustical Society of America, Vol. 42, No. 1, 1967, pp. 6-17. https://doi.org/10.1121/1.1910576

[5] Selamet, A., and Lee, I., "Helmholtz resonator with extended neck," The Journal of the Acoustical Society of America, Vol. 113, No. 4, 2003, pp. 1975-1985. https://doi.org/10.1121/1.1558379. URL https://doi.org/10.1121/1.1558379

[6] Simon, F., "Long elastic open neck acoustic resonator for low frequency absorption," Journal of Sound and Vibration, Vol. 421, 2018, pp. 1-16. https://doi.org/10.1016/j.jsv.2018.01.044.

[7] Guo, J., Fang, Y., Jiang, Z., and Zhang, X., "An investigation on noise attenuation by acoustic liner constructed by Helmholtz resonators with extended necks," The Journal of the Acoustical Society of America, Vol. 149, No. 1, 2021, pp. 70-81. https://doi.org/10.1121/10.0002990 URL https://doi.org/10.1121/10.0002990

[8] Guo, J., Zhou, T., Fang, Y., and Zhang, X., "Experimental study on a compact lined circular duct for small-scale propeller noise reduction," Applied Acoustics, Vol. 179, 2021, p. 108062. https://doi.org/https://doi.org/10.1016/j.apacoust.2021.108062 URL https://www.sciencedirect.com/science/article/pii/S0003682X21001559

[9] Howerton, B., and Jones, M., “A Conventional Liner Acoustic/Drag Interaction Benchmark Database," 2017. //doi.org/10.2514/6.2017-4190

[10] Jasinski, C., and Corke, T., "Mechanism for Increased Viscous Drag over Porous Sheet Acoustic Liners," AIAA Journal, Vol. 58, No. 8, 2020, pp. 3393-3404. https://doi.org/10.2514/1.J059039, URL https://doi.org/10.2514/1.J059039

[11] Zhang, Q., and Bodony, D. J., "Numerical investigation of a honeycomb liner grazed by laminar and turbulent boundary layers," Journal of Fluid Mechanics, Vol. 792, 2016, p. 936-980. https://doi.org/10.1017/jfm.2016.79

[12] Léon, O., Méry, F., Piot, E., and Conte, C., "Near-wall aerodynamic response of an acoustic liner to harmonic excitation with grazing flow," Experiments in Fluids, Vol. 60, No. 9, 2019, p. 144. https://doi.org/10.1007/s00348-019-2791-5.

[13] Elnady, T., Bodén, H., and Kontio, T., "Impedance of SDOF perforated liners at high temperatures," 10th AIAA/CEAS Aeroacoustics Conference, 2004, p. 2842.

[14] Giachetti, B., Fénot, M., Couton, D., and Plourde, F., "Influence of multi-perforation synthetic jet configuration on heat transfer enhancement," International Journal of Heat and Mass Transfer, Vol. 125, 2018, pp. 262-273. https://doi.org/https://doi.org/ 10.1016/j.ijheatmasstransfer.2018.04.073 URL https://www.sciencedirect.com/science/article/pii/S0017931017353425

[15] Esnault, S., Duchaine, F., Gicquel, L., and Moreau, S., "Large Eddy Simulation of Heat Transfer Within a Multi-Perforation Synthetic Jets Configuration,” Journal of Turbomachinery, Vol. 142, No. 6, 2020.

[16] Méry, F., Piot, E., Sebbane, D., Reulet, P., Simon, F., and Carazo Méndez, A., "Experimental Assessment of the Effect of Temperature Gradient Across an Aeroacoustic Liner," Journal of Aircraft, Vol. 56, No. 5, 2019, pp. $1809-1821$. https://doi.org/10.2514/1.C035157

[17] Lafont, V., Mery, F., Reulet, P., and Simon, F., "Surface temperature measurement of acoustic liners in the presence of grazing flow and thermal gradient," Experiments in Fluids, Vol. 62, 2021. https://doi.org/10.1007/s00348-021-03184-w

[18] Roncen, R., Méry, F., Piot, E., and Simon, F., "Statistical Inference Method for Liner Impedance Eduction with a Shear Grazing Flow," AIAA Journal, Vol. 57, No. 3, 2019, pp. 1055-1065. https://doi.org/10.2514/1.J057559

[19] Minotti, A., Simon, F., and Gantié, F., "Characterization of an acoustic liner by means of Laser Doppler Velocimetry in a subsonic flow," Aerospace Science and Technology, Vol. 12, No. 5, 2008, pp. 398-407. https://doi.org/10.1016/j.ast.2007.09.007. URL http://www.sciencedirect.com/science/article/pii/S1270963807001150.

[20] Colburn, A. P., "A method of correlating forced convection heat-transfer data and a comparison with fluid friction," International Journal of Heat and Mass Transfer, Vol. 7, No. 12, 1964, pp. 1359-1384. https://doi.org/https://doi.org/10.1016/00179310(64)90125-5. URL https://www.sciencedirect.com/science/article/pii/0017931064901255

[21] Chung, J. Y., and Blaser, D. A., "Transfer function method of measuring in-duct acoustic properties. I. Theory," The Journal of the Acoustical Society of America, Vol. 68, No. 3, 1980, pp. 907-913. https://doi.org/10.1121/1.384778. 
[22] Primus, J., Piot, E., and Simon, F., "An adjoint-based method for liner impedance eduction: Validation and numerical investigation," Journal of Sound and Vibration, Vol. 332, No. 1, 2013, pp. 58-75. https://doi.org/10.1016/j.jsv.2012.07.051. URL http://www.sciencedirect.com/science/article/pii/S0022460X12006268

[23] Lafont, V., Méry, F., Roncen, R., Simon, F., and Piot, E., "Liner Impedance Eduction Under Shear Grazing Flow at a High Sound Pressure Level," AIAA Journal, Vol. 58, No. 3, 2020, pp. 1107-1117. https://doi.org/10.2514/1.J058756 